\title{
PNCK depletion inhibits proliferation and induces apoptosis of human nasopharyngeal carcinoma cells in vitro and in vivo
}

\author{
Yuanji $\mathrm{Xu}^{1^{*}}$, Jiling Wang ${ }^{2 *}$, Shaoli Cai ${ }^{3,4}$, Guanghao Chen ${ }^{5}$, Nanyang Xiao ${ }^{3,4}$, Yajuan Fu ${ }^{3,4}$, Qi Chen ${ }^{3,4}$, Sufang
} $\mathrm{Qiu}^{1,6^{\boxplus}}$

1. Department of Radiation Oncology, Fujian Medical University Cancer Hospital \& Fujian Cancer Hospital, Fuzhou, China.

2. Department of Medical Oncology, The First Hospital of Putian City, Putian, China.

3. Biomedical Research Center of South China, Fujian Normal University, Fuzhou, China.

4. The Key Laboratories of Innate Immune Biology of Fujian Province, Fuzhou, China.

5. Longyan People Hospital, Longyan, China.

6. Fujian Provincial Key Laboratory of Translational Cancer Medicine, Fuzhou, China.

*These authors contributed equally to this paper.

$\triangle$ Corresponding author: Sufang Qiu, Department of radiation oncology, Fujian Medical University Cancer Hospital \& Fujian Cancer Hospital, Fuzhou, China; Fujian Provincial Key Laboratory of Translational Cancer Medicine, Fuzhou, China. E-mail: sufangqiu@fjmu.edu.cn

(C) The author(s). This is an open access article distributed under the terms of the Creative Commons Attribution License (https://creativecommons.org/licenses/by/4.0/). See http://ivyspring.com/terms for full terms and conditions.

Received: 2019.01.31; Accepted: 2019.10.26; Published: 2019.12.03

\begin{abstract}
Purpose: Recent studies indicate that pregnancy upregulated non-ubiquitous calmodulin kinase (PNCK) is significantly up-regulated in breast and renal carcinomas. However, the expression profile and its biological relevance of PNCK in nasopharyngeal carcinoma (NPC) have not been elucidated.

Methods: The expression level of PNCK was detected in specimens of NPC $(n=10)$ and normal tissues $(n=10)$ by real-time PCR and immunohistochemistry. Celigo Cell Counting and MTT assay were used to measure cell viability. Apoptosis was detected by flow cytometric analysis and caspases 3/7 activity assay. Real-time PCR and Western blotting were performed to evaluate the expression of PNCK. The bioluminescence imaging was used to evaluate the effects of PNCK knockdown on tumor growth using a xenograft animal model. The global gene expression profile was determined in wild type and PNCK-depleted CNE-2 cells via transcriptomics analysis. For mechanical investigation, the changes of PI3K/AKT/mTOR signaling pathway were detected by Western blotting.

Results: The mRNA and protein levels of PNCK were increased in human NPC samples. In vitro experiments showed that shRNA or CRISPR-Cas9 mediated silencing of PNCK inhibited proliferation and induced apoptosis in NPC cells. In addition, in vivo assay revealed that knockdown of PNCK suppressed tumor growth. Consistently, a significant reduction of tumor bioluminescence in mice inoculated with PNCK-knockdown cells compared to that of control cells. In gene expression, the transcriptomics analysis revealed that there were 589 upregulated genes and 589 downregulated genes in PNCK-knockdown cells. Ingenuity Pathway Analysis (IPA) identified significant changes of PI3K/AKT/mTOR signaling pathway in PNCK-knockdown cells. Furthermore, western blot analysis revealed that interference with PNCK reduced the phosphorylation levels of PI3K, AKT and mTOR in CNE-2 cells.

Conclusion: This study for the first time demonstrates that knockdown of PNCK could suppress growth and induce apoptosis of NPC cells both in vitro and in vivo by regulating PI3K/AKT/mTOR signaling pathway. These findings suggest that PNCK might be a novel therapeutic target for NPC treatment.
\end{abstract}

Key words: NPC, PNCK, gene expression profiling.

\section{Introduction}

Nasopharyngeal carcinoma (NPC) is a squamous epithelial malignant tumor arising from the lateral wall surface of nasopharynx with a high incidence in southern China, Japan and Southeast Asia [1]. Prevalence survey shows that the southern Chinese have the highest incidences of NPC in the world [2]. 
Genetic, environmental and microbial factors are thought to be involved in the carcinogenesis of NPC [3]. Due to the high sensitivity of NPC cells to ionizing radiation, radiotherapy is the main modality for treatment of NPC. However, some NPC patients have a poor prognosis because of no suitable biomarker for early detection and poor understanding of the molecular mechanisms [4-6]. Thus, the further understanding of the underlying mechanisms of NPC tumorigenesis will contribute to develop novel options for the diagnosis and therapy of NPC.

Calmodulin (CaM) kinases are a group of serine/threonine kinases that catalyze various biological processes, such as neurotransmitter release, muscle contraction, proliferation, differentiation, and apoptosis [7-9]. Pregnancy-upregulated nonubiquitous calmodulin kinase (PNCK) is a novel member of the CaM kinase family, which shares $45-70 \%$ sequence similarity with the kinase domain of the CaM kinase family. PNCK is widely expressed in the central nervous system and in a variety of tissues including heart, breast, brain, uterus, and stomach [10]. Recent studies have found that PNCK play critical roles in cytoplasmic and nuclear signal transduction, thereby regulating various biological processes [11,12]. However, the molecular mechanism by which PNCK contributes to the malignant NPC remains largely unknown.

Therefore, it is critical to elucidate the mechanism by which PNCK exerts its functional role in the progression of NPC. In the present study, for the first time, we explored the functional relevance of PNCK using NPC tissue samples, cell lines and animal models. For mechanical investigation, transcriptome analysis was performed and many signaling pathways were found to be altered. Analysis of clinical samples revealed a significant elevation of PNCK in NPC. Moreover, in vitro and in vivo studies showed that knockdown of PNCK substantially inhibited growth and induced apoptosis in human NPC cells. In addition, transcriptomic analysis revealed that $\mathrm{PI} 3 \mathrm{~K} / \mathrm{AKT} / \mathrm{mTOR}$ pathway was remarkably changed, which may be responsible for PNCK-mediated cellular behaviors. Taken together, our study indicates that the PNCK could be a target for treatment of NPC.

\section{Materials and methods}

\section{Patients and tissue specimens}

In this study, 8 freshly frozen NPC and 10 normal nasopharyngeal tissue were collected in Fujian Cancer Hospital between January 2017 and March 2017. Then, paraffin-embedded specimens of NPC $(n=10)$ and normal tissues $(n=10)$ were used for gene expression analysis. These patients had no radiotherapy or chemotherapy history before biopsy. NPC was pathologically confirmed by two senior pathologists who were blinded to the clinical information of patients. This study was approved by the Institute Research Medical Ethics Committee of Fujian Cancer Hospital, Fujian Medical University Cancer Hospital (\#2017-051-01), with a written consent form signed by patients.

\section{Cell culture}

The human NPC cell lines (CNE-2, CNE-1 and $5-8 \mathrm{~F})$ were purchased from the Cell Resource Center (Shanghai Institutes for Biological Sciences, China Academy of Sciences). NPC C666-1 cell line was a gift form Prof. Geoge S.W. Tsao of the University of Hong Kong. Cells were cultured in Dulbecco's Modified Eagle Medium supplemented with 10\% FBS, 100 $\mathrm{U} / \mathrm{mL}$ penicillin, and $100 \mathrm{U} / \mathrm{mL}$ streptomycin, and were maintained at $37^{\circ} \mathrm{C}$ in $5 \% \mathrm{CO}_{2}$ incubator.

\section{Transcriptome analysis}

Total RNAs were extracted using TRIZOL Reagent (Life technologies, Carlsbad, CA, USA) following the manufacturer's instructions and checked for RNA integrity by an Agilent Bioanalyzer 2100 (Agilent technology, Santa Clara, CA, USA). Qualified total RNA was further purified by RNeasy microkit (QIAGEN, GmBH, Germany) and RNase Free DNase Set (QIAGEN, GmBH, Germany). Total RNAs were amplified, labeled and purified by using GeneChip 3'IVT Express Kit (Affymetrix, Santa Clara, CA, USA) followed the manufacturer's instructions to obtain biotin labeled RNA. Array hybridization and wash was performed using GeneChip ${ }^{\circledR}$ Hybridization, wash and stain Kit (Affymetrix, Santa Clara, CA) in Hybridization Oven 645 (Affymetrix, Santa Clara, CA) and Fluidics Station 450 (Affymetrix, Santa Clara, CA) followed the manufacturer's instructions. Slides were scanned by GeneChip ${ }^{\circledR}$ Scanner 3000 (Affymetrix, Santa Clara, CA, US) and Command Console Software 3.1 (Affymetrix, Santa Clara, CA, US) with default settings. Differentially expressed genes with statistical significance, a fold change filtering between two samples was performed and the default threshold was $\geq 1.5$ fold-change. The biological processes were identified using Ingenuity Pathway Analysis (http://www.ingenuity.com/ products/ipa).

\section{Cell proliferation assay}

Cell proliferation was determined using MTT [3-(4, 5-dimethylthiazol-2-yl)-2, 5-diphenyl tetrazolium bromide] assay (Roche Diagnosis). Briefly, cells were plated into 96-well plates at the density of 2,000 cells/well in triplicates and cultured 
in DMEM supplemented with 10\% FBS. After $24 \mathrm{~h}$ incubation at $37^{\circ} \mathrm{C}, 20 \mu \mathrm{l}$ of $5 \mathrm{mg} / \mathrm{ml}$ MTT was added and further cultured for 4 hours. After discard of culture media, $100 \mu \mathrm{l} /$ well of dimethyl sulfoxide was added to well and the optical density was measured at $490 \mathrm{~nm}$ using a Microplate Reader (Bio-Rad, Hercules, CA, USA). All experiments were performed at least three times.

\section{Apoptosis assay}

Cells were collected by trypsinization, washed twice with PBS and fixed in $80 \%$ ice-cold ethanol in PBS. Then, cells $\left(\geq 5 \times 10^{5}\right)$ were re-suspended in $200 \mu \mathrm{l}$ binding buffer and incubated with $10 \mu \mathrm{l}$ staining solution containing FITC-conjugated annexin V antibody (cat: 88-8007, eBioscience, CA, USA). After incubation for $15 \mathrm{~min}$ at room temperature in the dark the apoptotic cells were detected by flow cytometer following the manufacturer's instruction.

\section{Caspase 3/7 activity assay}

The Caspase $3 / 7$ activity was determined in each group using a commercial kit (Caspase-Glo® 3/7 Assay, Promega, Shanghai) according to the manufacturer's instruction. In brief, $100 \mu 1$ Caspase3/7 reagent was added to each well of the plates and incubated at room temperature for $2 \mathrm{~h}$. Absorbance values were detected using a microplate reader at A405nm (Tecan infinite, Mannedorf, Switzerland).

\section{Immunohistochemical staining}

The paraffin-embedded blocks were cut into $3-\mu \mathrm{m}$ thickness sections and mounted on glass slides coated with poly-L-lysine, deparaffinized with xylene and rehydrated with gradient ethanol. The slides were heated and incubated in a $10 \mathrm{mM}$ citrate buffer. After inactivating endogenous peroxidase by $\mathrm{H}_{2} \mathrm{O}_{2}$, the slides were incubated with primary antibody targeting PNCK (1:20, HPA007458, Sigma, USA) for 1 $\mathrm{h}$ followed by incubation with biotinylated secondary antibody. The staining intensity was considered as negative (0), weak (1), moderate (2), or strong (3). The percentage of positive staining was scored as $0 \%(0)$, $<25 \%$ (1), $25-50 \%$ (2), $50-75 \%$ (3), or $>75 \%$ (4). The immunostainings of PNCK was evaluated by two pathologists without knowledge of patient characteristics and a consensus was provided on staining patterns.

\section{Real time PCR}

Total RNA from cells or tissues was extracted using Trizol reagent (Life Technologies), and reversely transcribed into cDNA. The expression levels of indicated genes were measured by real time PCR on ABI 7700 system using the following primers: forward PNCK-F: 5'-TGACATCTCAGAATCAGCCA
AAG-3', reverse PNCK-R 5'-GTGTCCGAGCAA AGTTCTTCC-3'; GAPDH-F: 5' - TGACTTCAACAGC GACACCCA-3', reverse GAPDH-R 5'- CACCCTGT TGCTGTAGCCAAA-3'. PCR was conducted with the following parameters: an initial denaturation step of 30 seconds at $95^{\circ} \mathrm{C}$; and $94^{\circ} \mathrm{C}$ (30 seconds), $58^{\circ} \mathrm{C}(30$ seconds), $72^{\circ} \mathrm{C}$ (50 seconds) in a total 35 cycles with a final extension step at $72^{\circ} \mathrm{C}$ for $5 \mathrm{~min}$. All samples were normalized to GAPDH and expression fold changes were calculated using $2-\Delta \Delta \mathrm{Ct}$ methods.

\section{Western blotting}

Cells were collected, lysed in RIRA lysis buffer, and subject to centrifugation at $12,000 \mathrm{rpm}$ for $10 \mathrm{~min}$. The protein concentration was determined using a BCA protein assay kit (Beyotime, Haimen, China). Equal amounts of protein lysates were separated by denaturing sodium dodecyl sulfate-polyacrylamide gels and transferred to nitrocellulose membranes (Bio-Rad Laboratories, Hercules, CA). After blocking in $5 \%$ nonfat dry milk, the membranes were incubated with primary antibodies targeting PNCK (1:500, Sigma, USA), PI3K (1:1000, Cell Signaling Technology, USA), AKT (1:1000, Cell Signaling Technology, USA), mTOR (1:1000, Cell Signaling Technology, USA), p-PI3K (1:1000, Abcam, USA), p-AKT (1:2000, Cell Signaling Technology, USA), p-mTOR (1:5000, Cell Signaling Technology, USA) and GAPDH (1:1000, Santa Cruz, USA) at $4^{\circ} \mathrm{C}$ overnight. The membranes were rinsed with PBST (PBS with 0.1\% Tween-20) three times and incubated with the appropriate secondary antibodies for $1 \mathrm{~h}$ at room temperature. The protein bands were detected using an enhanced chemiluminescence kit (GE Healthcare, Piscataway, NJ).

\section{Xenograft model}

Female athymic BALB/c mice (4-6 weeks old) were purchased from Shanghai Slac Laboratory Animal Co. Ltd. (Shanghai, China). All procedures for animal studies were approved by Animal Care and Use Committee of Fujian Cancer Hospital. Wild-type or PNCK-knockdown CNE-2 cells were injected subcutaneously into nude mice ( $\mathrm{n}=10$ per group). After 10 days, tumors were measured with calipers once a week for five weeks. Tumor volume was calculated according to the following formula: $\mathrm{V}=$ $\Pi / 6 \times \mathrm{L} \times \mathrm{W}^{2}$ where $\mathrm{V}=$ volume $\left(\mathrm{mm}^{3}\right), \mathrm{L}=$ length $(\mathrm{mm})$, and $\mathrm{W}=$ width $(\mathrm{mm})$ [13]. At the end of experiments, mice were euthanized and tumors were excised and weighed. For measurement of bioluminescence imaging density, mice were anesthetized with isoflurane gas and injected intraperitoneally with $150 \mathrm{mg} / \mathrm{kg}$ D-luciferin aqueous solution. The images were captured $10 \mathrm{~min}$ following 
injection using an IVIS 50 Imaging system (Caliper Life Sciences, Alameda, CA, USA). Signal intensity was quantified within a region of interest.

\section{Statistical analysis} SPSS 17.0. The data were presented as the mean \pm standard deviation (S.D.) of at least three independent experiments. Differences between groups were compared using Student's $t$ test or ANOVA. All data were considered significant difference if $\mathrm{P}$ values were $<0.05$.

\section{Results}

\section{Up-regulation of PNCK in human NPC} NPC cancerous and normal tissues were determined by real-time PCR and immunohistochemical staining. The results (Fig 1A and B) showed that the mRNA and protein levels of PNCK were higher in the NPC tissues compared to the normal tissues. Furthermore, Figure 1C showed that PNCK in four NPC cell lines (CNE-2, C666-1, CNE-1 and 5-8F) was detectable, indicating that PNCK is commonly possessed by NPC cells.

\section{ShRNA-mediated PNCK knockdown inhibited growth and induced apoptosis in NPC cells}

All statistical analyses were performed with

Firstly, mRNA and protein levels of PNCK in

To investigate the role of PNCK in the growth of
A

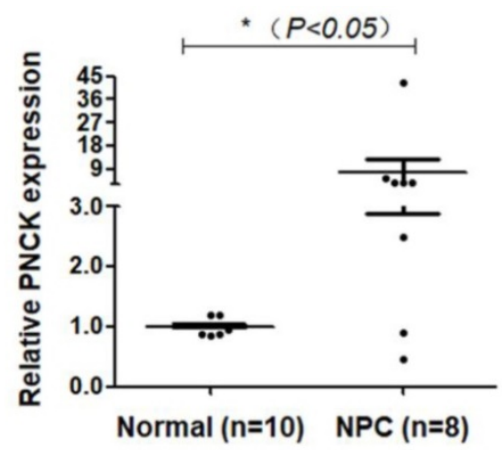

NPC cells, we knocked-down the expression of PNCK by shRNA in CNE-2 cells (Figure 2A) at the mRNA and protein levels as confirmed by real-time PCR and western blot analysis (Figure 2B). The growth and proliferation of PNCK knocked-down cells was inhibited compared to control cells demonstrated by Celigo Cell Counting (Figure 2C) and MTT assays (Figure 2D). Furthermore, the PNCK knockdown could enhance the programmed cell death (apoptosis), as showed in Annexin V-FCM assay (Figure 2E). Moreover, the caspase $3 / 7$ activity in PNCK knockdown tumor cells was elevated in ELISA assay (Figure 2F).

\section{CRISPR-Cas9-mediated PNCK knockout induced apoptosis in NPC cells}

To completely deplete PNCK, CNE-2 cells were co-transfected with CRISPR-Cas9 and sgRNA against the PNCK (Figure 3A). Western blot analysis showed that PNCK was completely silenced by CRISPR/Cas9-mediated gene knockout (Figure 3B). In addition, ELISA assay showed that PNCK knockout significantly increased the caspase 3/7 activity in CNE-2 cells (Figure 3C). Consistently, flow cytometric analysis revealed an elevated apoptosis in PNCK knockout CNE-2 cells (Figure 3D). Collectively, these data indicated that knockout of PNCK induced apoptosis in NPC cells.

C

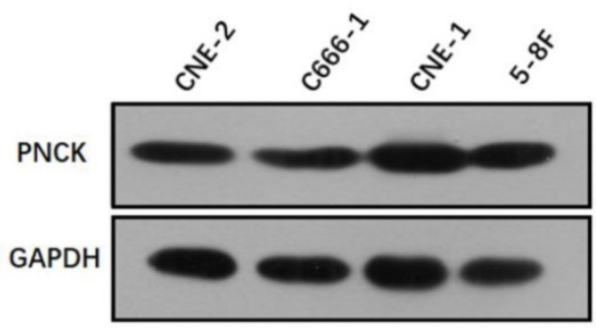

B
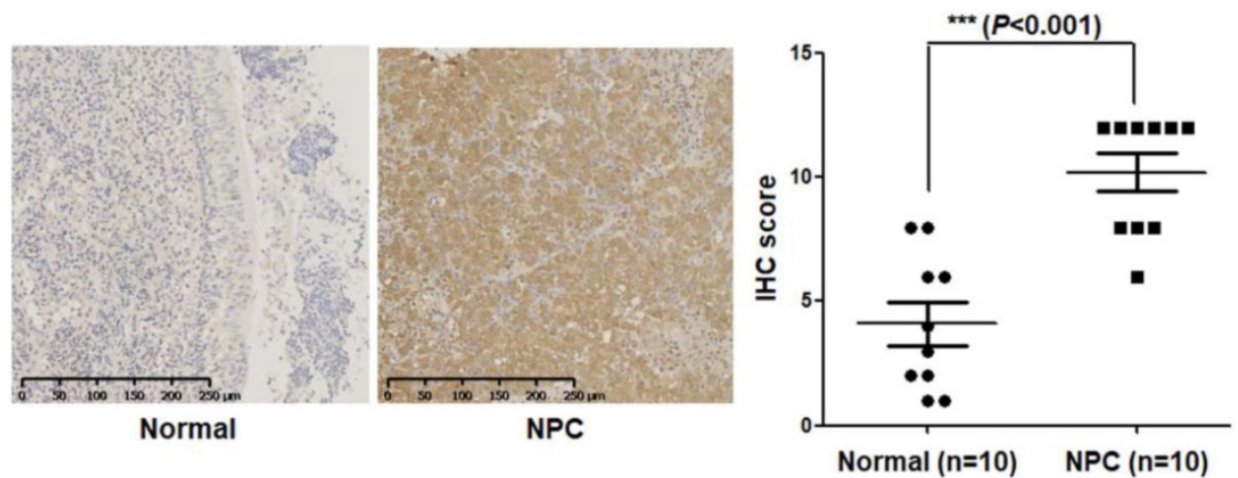

Figure 1. Up-regulation of PNCK in human NPC. The expression of PNCK in NPC tumor tissues and the matched adjacent nasopharyngeal tissues was detected by real time PCR (A) and immunohistochemical staining (B). (C) Western blot was performed to detect the expression of PNCK in four NPC cell lines including CNE-2, C666-1, CNE-1 and 5-8F. 
A

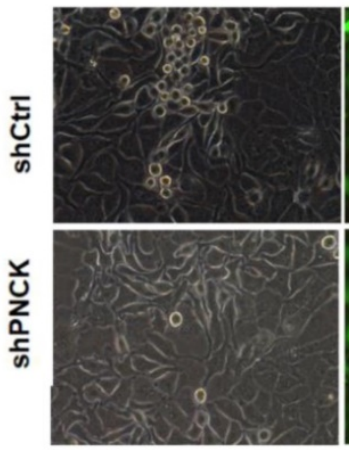

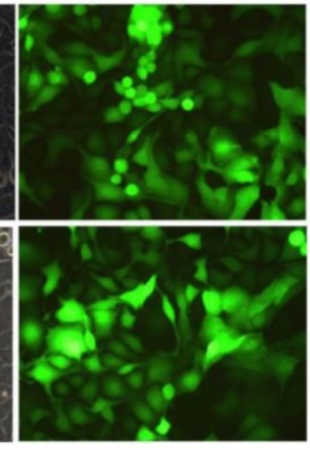

B
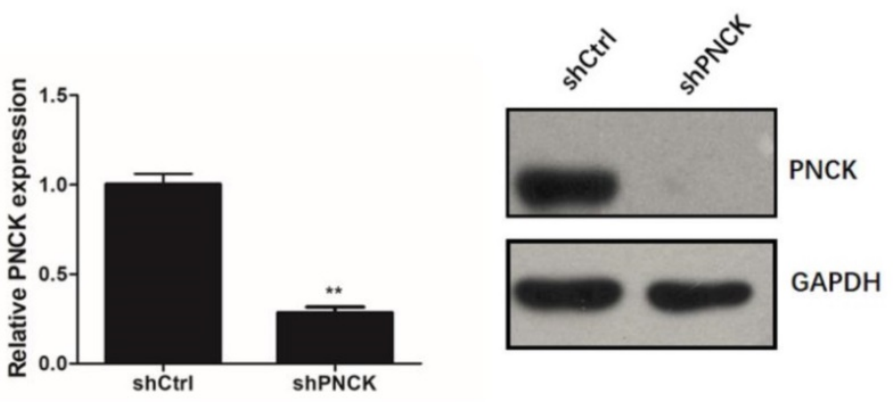

D

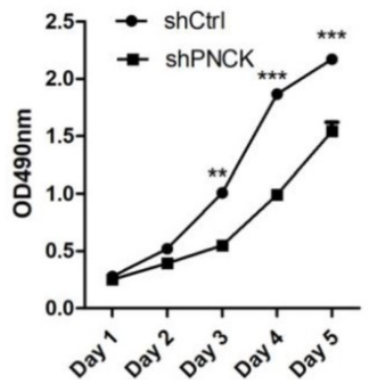

F
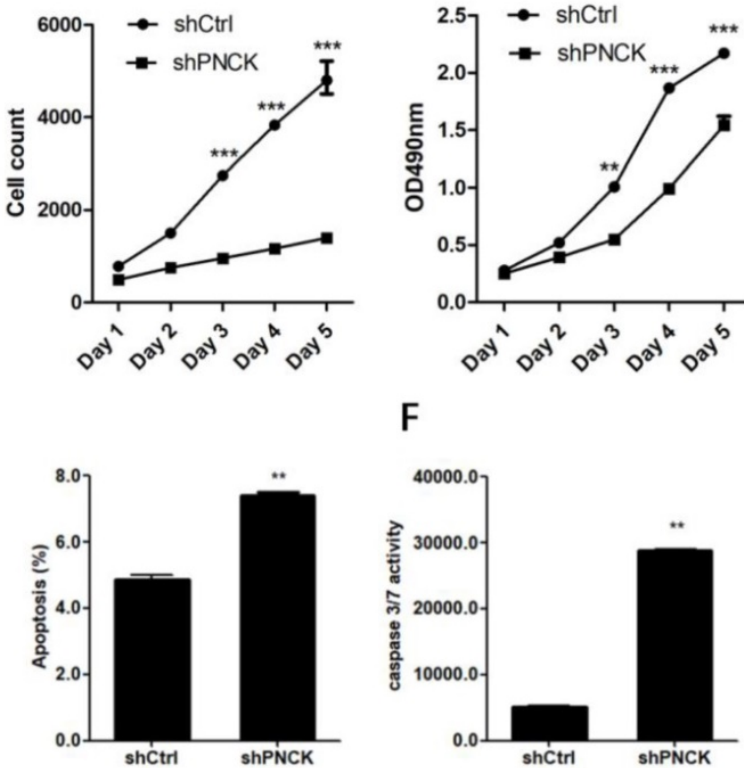

Figure 2. ShRNA-mediated PNCK knockdown inhibited growth and induced apoptosis in NPC cells. Human NPC CNE-2 cells were infected with PNCK shRNA using a lentivirus-GFP system (A). After $72 \mathrm{~h}$, silencing of PNCK expression was validated by using real time PCR (B) and western blot analysis (C). Celigo Cell Counting (C) and MTT assays (D) were conducted to measure the effect of PNCK knockdown on cell viability. The effect of PNCK knockdown on cell apoptosis was assessed by determining the apoptotic cell death $(\mathrm{E})$ and caspase $3 / 7$ activity $(\mathrm{F})$. $* * \mathrm{P}<0.01, * * * \mathrm{P}<0.001$.

\section{In vivo effect of PNCK knockdown on NPC cell growth}

To explore if the in vitro effect of PNCK-knockdown can be translated in vivo, the PNCK knockdown CNE-2 cells were injected subcutaneously into the flank of BABL/c nude mice. After 2 weeks later, the tumor volume in each mouse was measured once a week for consecutive 5 weeks. There was a significant reduction of tumor volume and bioluminescence imaging (BLI) signals in tumors formed by PNCK-knockdown CNE-2 cells compared to those formed by control cells (Figure 4A and B). Similarly, the lack of PNCK significantly inhibited the tumor weight tumors at the end of this study as compared with the tumors formed by wild-type NPC cells (Figure 4C). Collectively, these data indicate that inhibition of PNCK exhibits anti-proliferative effect in in vivo model of xenograft nude mice.

\section{Effects of PNCK depletion on PI3K/AKT/mTOR signaling pathway}

Subsequently, genome-wide expression profiling was conducted to determine the differentially expressed genes in the CNE-2 cell line upon PNCK knockdown. Total RNAs were extracted from the wild type or PNCK-knockdown CNE-2 cells and subjected to microarray analysis using Affymetrix GeneChip PrimeView Human Gene Expression Arrays. The purity (A260/280 value) of all RNA samples prepared was $>1.95$ (Table 1 ).

Transcriptomic analysis revealed 589 genes were up-regulated and 589 genes were down-regulated (fold change $>=1.5$, P-value $<0.05$ ). Volcano plots and 
heat maps of these differentially expressed genes visualized a clear distinction between wild type and PNCK knockdown CNE-2 cells (Figure 5A and B). Moreover, pathway analysis using Ingenuity Pathway Analysis (IPA) revealed significant changes of PI3K/AKT/mTOR signaling pathway. Furthermore, western blot analysis showed that interference with

A B
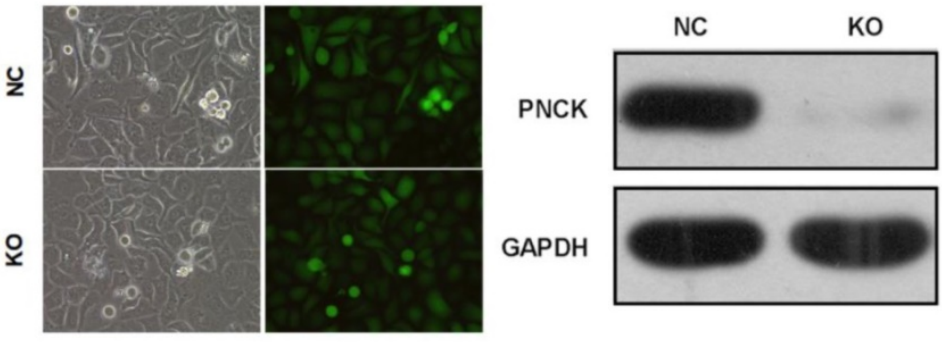

D

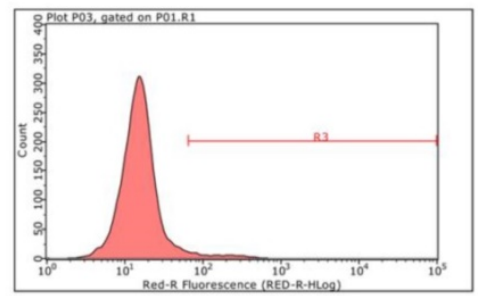

NC

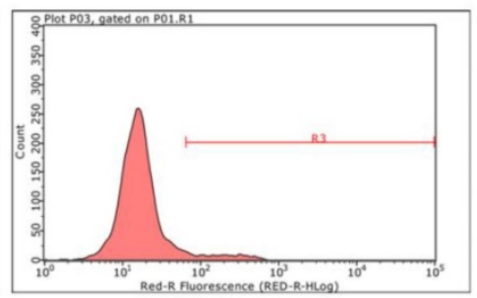

KO
PNCK expression reduced the phosphorylation levels of PI3K, AKT and mTOR in CNE-2 cells (Figure 5C). There were no significant changes of total PI3K, AKT and mTOR between control and shPNCK groups. Collectively, these findings reveal that depletion of PNCK inhibits PI3K/AKT/mTOR signaling pathway in NPC cells.

C
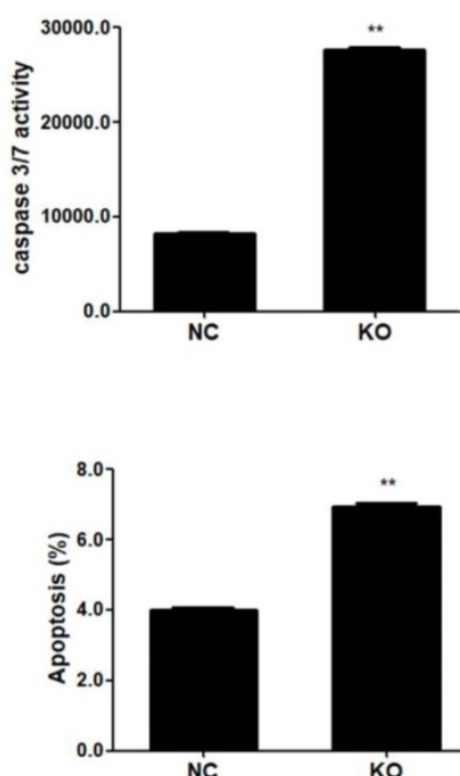

Figure 3. CRISPR-Cas9-mediated PNCK knockout induced apoptosis in NPC cells. Human NPC CNE-2 cells were co-transfected with CRISPR-Cas 9 and sgRNA against the PNCK (A). After $72 \mathrm{~h}$, silencing of PNCK expression was validated by using western blot analysis (B). The effect of depletion of PNCK on cell apoptosis was assessed by determining the caspase $3 / 7$ activity $(C)$ and apoptotic cell death (D). $* * P<0.01$.

A

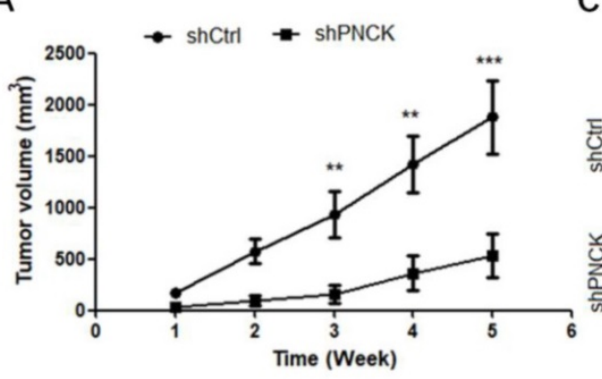

C

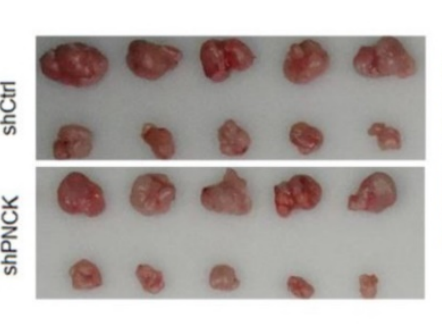

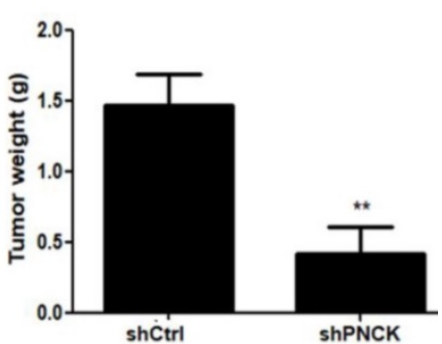

B
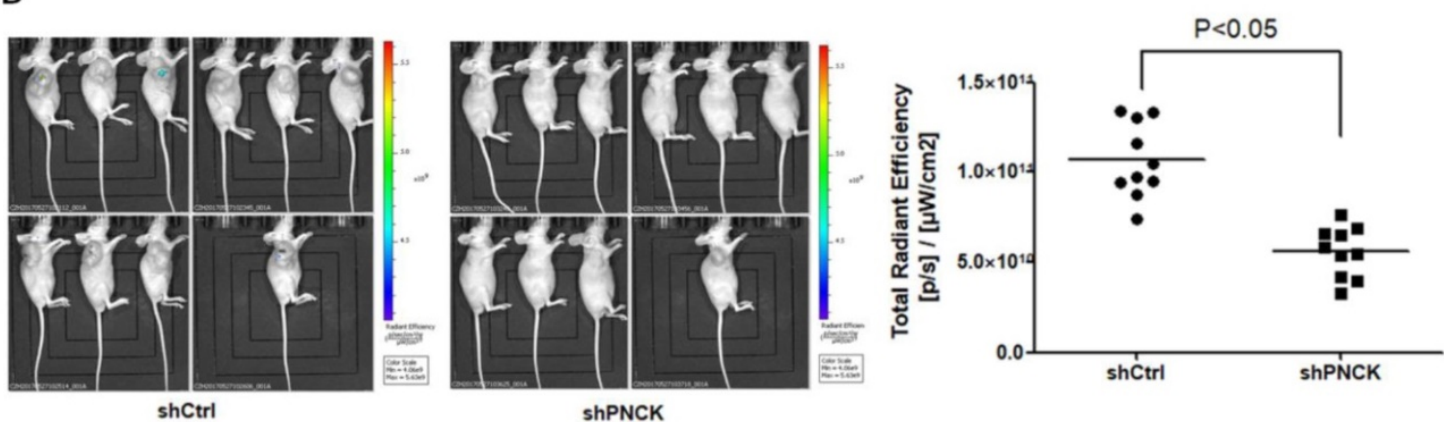

Figure 4. Depletion of PNCK inhibited tumor growth in vivo. The wild-type or PNCK-knockdown CNE-2 cells were injected subcutaneously into the flank of nude mice. Tumor diameters were measured at a regular interval of one week for up to five weeks, and the tumor volume was calculated (A). (B) In vivo bioluminescence imaging of tumors in wild-type or PNCK-knockdown CNE-2 tumor bearing mice. The xenografts were harvested after five weeks. The pictures of the tumors were taken, and the weights of the tumors were analyzed $(C)$. ** $P<0.01, * * * P<0.001$. 
A

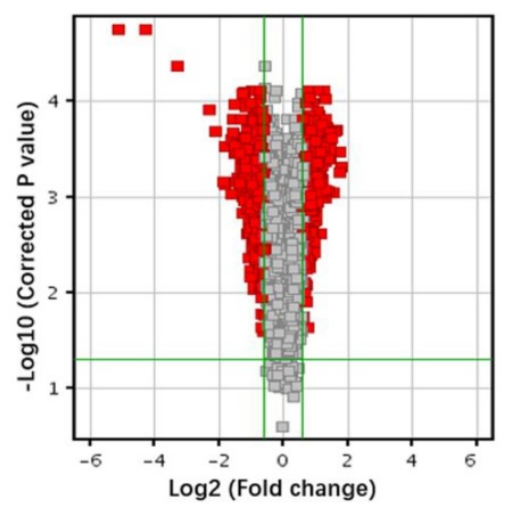

B

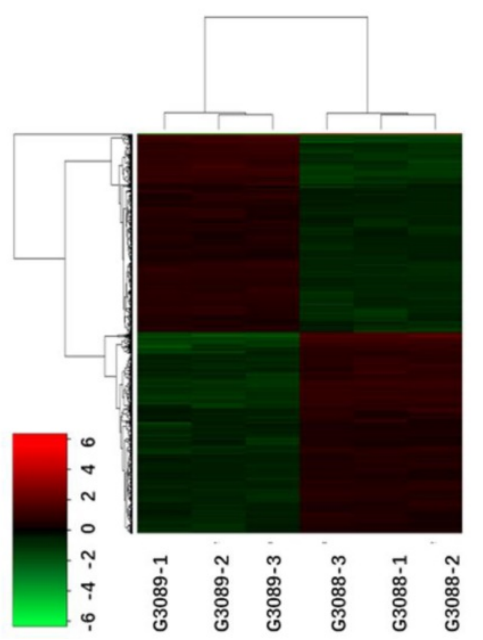

C

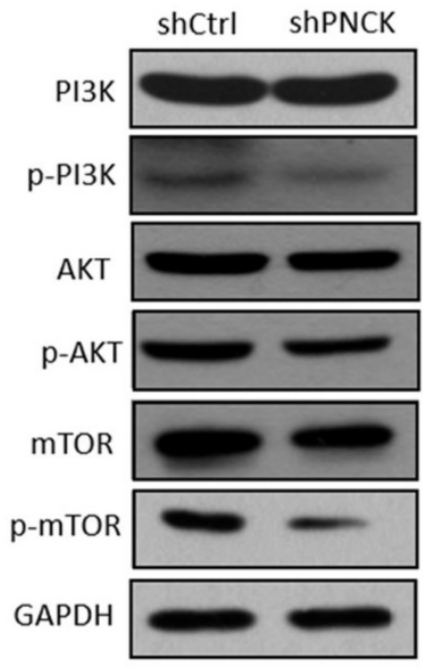

Figure 5. Effects of PNCK depletion on PI3K/AKT/mTOR signaling pathway. Volcano plots (A) and cluster analysis (B) in NPC cells after depletion of PNCK. (C) The expression of PI3K, AKT and mTOR and their phosphorylation levels in NPC cells were evaluated by western blot.

Table 1. Preparation of RNAs for microarray analysis*.

\begin{tabular}{llll}
\hline Sample number & Name & \multicolumn{2}{l}{ Thermo NanoDrop 2000 } \\
\cline { 3 - 4 } & & Concentration $(\mathrm{ng} / \mu \mathrm{L})$ & A260/A280 \\
\hline G3088-1 & NC & 491.8 & 1.96 \\
G3088-2 & NC & 505.6 & 1.97 \\
G3088-3 & NC & 522.8 & 2.02 \\
G3089-1 & KD & 491.1 & 1.97 \\
G3089-2 & KD & 485.5 & 1.97 \\
G3089-3 & KD & 500.1 & 1.97
\end{tabular}

*NC: control wild-type CNE-2 cells; KD: PNCK knockdown CNE-2 cells. A total of six samples were subjected to microarray analysis with three samples in each group.

\section{Discussion}

In this study, the high expression of PNCK was detected in NPC tumor tissues and NPC cell lines. Silencing of PNCK inhibited cell proliferation and induced apoptosis in vitro. Moreover, animal experiments revealed that PNCK knockdown suppressed tumor growth. In addition, transcriptomic analysis indicated substantial changes in the gene expression and various signaling pathways involved in tumor growth and development. We conclude that PNCK plays a potential oncogenic role in NPCs by promoting NPC cell proliferation and tumor growth, a new insight into understanding the mechanisms of NPC tumorigenesis and exploring the potential therapeutic targets for NPCs.

PNCK, a newly discovered CaM kinase, is elevated in the mouse mammary gland during pregnancy in a subset of epithelial cells [10]. Similarly, PNCK is up-regulated in the transformation of human breast cancer, suggesting that PNCK may be involved in mammary development and carcinogenesis [11]. In addition, high expression of PNCK is reported in clear cell renal cell carcinoma (ccRCC), which is correlated with shorter overall survival, an independent prognostic factor for survival in ccRCC [14]. Recently, studies have revealed that PNCK induces ligand-independent EGFR degradation by probable perturbation of the Hsp90 chaperone complex, representing an attractive target in EGFR-regulated oncogenesis [15-17]. Additionally, ectopic expression of PNCK causes trastuzumab resistance in HER-2 amplified breast cancer via PTEN-mediated process, suggesting that inhibition of PNCK may be a novel strategy to overcome drug resistance [18].

Gene expression profiling, pathway analysis and IPA analysis by comparison of with PNCK-depleted NPC cells with its wild-type cells revealed that hundreds of genes were significantly altered after PNCK knockdown, especially the signaling pathways (i.e., PI3K/AKT/mTOR, IL-1 and integrin signaling) and biological events (i.e., cell deaths and survival, apoptosis, cancer pathogenesis, cellular development). The PI3K/AKT/mTOR pathway is a survival pathway constitutively activated in many types of cancer. This pathway is considered as an attractive therapeutic target for cancer treatment because it serves as a convergence point, controlling the multiple biological processes that contribute to the initiation and progression of cancer $[22,23]$. It has been reported that blocking PI3K/AKT signaling attenuates metastasis of NPC cells by inducing mesenchymal-epithelial reverting transition [24]. Moreover, genetic variations in the $\mathrm{PI} 3 \mathrm{~K} / \mathrm{AKT} / \mathrm{mTOR}$ pathway are suggested to be involved in the distant metastasis in NPC patients [25]. Accordingly, many studies have performed the preclinical evaluation of small-molecule inhibitors that target PI3K-mTOR as a novel therapeutic drug in NPC [26-28]. Consistently, our study validated that 
interference with PNCK expression reduced the phosphorylation levels of PI3K, AKT and mTOR, which contributed to the inhibition of $\mathrm{PI} 3 \mathrm{~K} / \mathrm{AKT} / \mathrm{mTOR}$ signaling pathway in NPC cells.

However, there are several limitations of this study, such as small sample size and lack of follow-up data that could have provided possible association between PNCK expression and clinicopathologic characteristics such as TNM stage, age, gender and survival. Although we found that PI3K/AKT/mTOR pathway is regulated by PNCK, the detailed regulation mechanisms are not understood. Therefore, a further investigation into the underlying molecular interaction between PNCK and $\mathrm{PI} 3 \mathrm{~K} / \mathrm{AKT} / \mathrm{mTOR}$ in NPC is needed.

In conclusion, the present study, for the first time, demonstrated the up-regulation of PNCK in NPC. Interference with PNCK expression exhibited an anti-oncogenic role in NPC cells both in vitro and in vivo. Moreover, transcriptomic analysis revealed that PNCK depletion induced substantial changes of gene expression and transduction signals. These findings indicate that PNCK might be a potential therapeutic target of NPC.

\section{Acknowledgements}

The project was funded by the grants from Fujian Provincial Health and Family Planning Commission Research Talent Training Program (\#2018-CX-13, \#2019-ZQN-14), Joint Funds for the Innovation of Science and Technology, Fujian Province (\#2018Y9105), Startup Fund for scientific research, Fujian Medical University (\#2018QH1222), the Natural Science Foundation of Fujian Province, China (\#2019J05140, \#2019J01201) and Fujian Provincial Key Laboratory of Translational Cancer Medicine and Science and Technology Program of Fujian Province, China (\#2018Y2003). The funders had no role in study design, data collection and analysis, decision to publish, or preparation of the manuscript.

\section{Competing Interests}

The authors have declared that no competing interest exists.

\section{References}

1. Siegel RL, Miller KD, Jemal A. Cancer statistics, 2018. CA Cancer J Clin. 2018;68:7-30.

2 Lee $\mathrm{AW}, \mathrm{Ma} \mathrm{BB}, \mathrm{Ng} \mathrm{WT}$, et al. Management of nasopharyngeal carcinomaNPC: Current practice and future perspective. J Clin Oncol. 2015;33:3356-64

3. Qu S, Liang ZG, Zhu XD. Advances and challenges in intensity-modulated radiotherapy for nasopharyngeal carcinoma. Asian Pac J Cancer Prev. 2015;16:1687-92.

4. Ooft ML, Braunius WW, Heus P, et al. Prognostic significance of the EGFR pathway in nasopharyngeal carcinoma: A systematic review and meta-analysis. Biomark Med. 2015;9:997-1010.
5. Janvilisri T. Omics-based identification of biomarkers for nasopharyngeal carcinomaNPC. Dis Markers. 2015;2015:762128.

6. Xu T, Tang J, Gu M, et al. Recurrent nasopharyngeal carcinoma: A clinical dilemma and challenge. Curr Oncol. 2013:20:406-19.

7. Nussinov R, Muratcioglu S, Tsai CJ, et al. The key role of calmodulin in KRAS-Driven adenocarcinomas. Mol Cancer Res. 2015;13:1265-73.

8. Berchtold MW, Villalobo A. The many faces of calmodulin in cell proliferation, programmed cell death, autophagy, and cancer. Biochim Biophys Acta. 2014;1843:398-435

9. Monaco S, Rusciano MR, Maione AS, et al. A novel crosstalk between calcium/calmodulin kinases II and IV regulates cell proliferation in myeloid leukemia cells. Cell Signal. 2015;27:204-14.

10. Gardner HP, Rajan JV, Ha SI, et al. Cloning, characterization, and chromosomal localization of Pnck, a $\mathrm{Ca}(2+)$ /calmodulin-dependent protein kinase. Genomics. 2000;63:279-88.

11. Gardner HP, Ha SI, Reynolds C, et al. The caM kinase, Pnck, is spatially and temporally regulated during murine mammary gland development and may identify an epithelial cell subtype involved in breast cancer. Cancer Res. 2000;60:5571-77

12. Burnik PT, Vrtacnik BE, Maver A, et al. Transcriptomic analysis and Meta-Analysis of human granulosa and cumulus cells. Plos One. 2015;10:136473.

13. Vizoso M, Ferreira HJ, Lopez-Serra P, et al. Epigenetic activation of a cryptic TBC1D16 transcript enhances melanoma progression by targeting EGFR. Nat Med. 2015;21:741-50.

14. Wu S, Lv Z, Wang $Y$, et al. Increased expression of pregnancy up-regulated non-ubiquitous calmodulin kinase is associated with poor prognosis in clear cell renal cell carcinoma. Plos One. 2013:8:59936.

15. Deb TB, Coticchia CM, Barndt R, et al. Pregnancy-upregulated nonubiquitous calmodulin kinase induces ligand-independent EGFR degradation. Am J Physiol Cell Physiol. 2008;295:365-77.

16. Deb TB, Zuo AH, Wang $\mathrm{Y}$, et al. Pnck induces ligand-independent EGFR degradation by probable perturbation of the Hsp90 chaperone complex. Am J Physiol Cell Physiol. 2011;300:1139-54.

17. Deb TB, Barndt RJ, Zuo AH, et al. PTEN-mediated ERK1/2 inhibition and paradoxical cellular proliferation following Pnck overexpression. Cell Cycle. 2014;13:961-73.

18. Deb TB, Zuo AH, Barndt RJ, et al. Pnck overexpression in HER-2 gene-amplified breast cancer causes Trastuzumab resistance through a paradoxical PTEN-mediated process. Breast Cancer Res Treat. 2015;150:347-61.

19. Rehemtulla A, Stegman LD, Cardozo SJ, et al. Rapid and quantitative assessment of cancer treatment response using in vivo bioluminescence imaging. Neoplasia. 2000;2:491-5

20. Soling A, Rainov NG. Bioluminescence imaging in vivo - application to cancer research. Expert Opin Biol Ther. 2003:3:1163-72.

21. Gabay C, Lamacchia C, Palmer G. IL-1 pathways in inflammation and human diseases. Nat Rev Rheumatol. 2010;6:232-41.

22. LoPiccolo J, Blumenthal GM, Bernstein WB, et al. Targeting the PI3K/Akt/mTOR pathway: Effective combinations and clinical considerations. Drug Resist Updat. 2008;11:32-50.

23. Yu JS, Cui W. Proliferation, survival and metabolism: The role of $\mathrm{PI} 3 \mathrm{~K} / \mathrm{AKT} / \mathrm{mTOR}$ signalling in pluripotency and cell fate determination. Development. 2016;143:3050-60.

24. Jiang $\mathrm{H}$, Gao $M$, Shen $\mathrm{Z}$, et al. Blocking PI3K/Akt signaling attenuates metastasis of nasopharyngeal carcinoma cells through induction of mesenchymal-epithelial reverting transition. Oncol Rep. 2014;32:559-66.

25. Guo Q, Lu T, Chen Y, et al. Genetic variations in the PI3K-PTEN-AKT-mTOR pathway are associated with distant metastasis in nasopharyngeal carcinoma patients treated with intensity-modulated radiation therapy. Sci Rep. 2016;6:37576.

26. Wong $\mathrm{CH}$, Loong $\mathrm{HH}$, Hui $\mathrm{CW}$, et al. Preclinical evaluation of the PI3K-mTOR dual inhibitor PF-04691502 as a novel therapeutic drug in nasopharyngeal carcinoma. Invest New Drugs. 2013;31:1399-408.

27. Liu T, Sun Q, Li Q, et al. Dual PI3K/mTOR inhibitors, GSK2126458 and PKI-587, suppress tumor progression and increase radiosensitivity in nasopharyngeal carcinoma. Mol Cancer Ther 2015;14:429-39.

28. Ma BB, Lui VW, Hui CW, et al. Preclinical evaluation of the mTOR-PI3K inhibitor BEZ235 in nasopharyngeal cancer models. Cancer Lett 2014;343:24-32. 